\title{
Synthesis by Reversed Phase Transfer Catalysis and Characterization of Naphthol AS-D Pigment
}

\author{
Eleonora Cornelia Crasmareanu, ${ }^{1}$ Vasile Simulescu, ${ }^{1,2}$ and Gheorghe Ilia ${ }^{1,3}$ \\ ${ }^{1}$ Institute of Chemistry Timisoara of the Romanian Academy, 24 Mihai Viteazu Boulevard, 300223 Timisoara, Romania \\ ${ }^{2}$ Centre for Materials Research, Faculty of Chemistry, Brno University of Technology, Purkyñova 118, 61200 Brno, Czech Republic \\ ${ }^{3}$ West University of Timisoara, 16 Pestalozzi Street, 300115 Timisoara, Romania \\ Correspondence should be addressed to Gheorghe Ilia; gheilia@yahoo.com
}

Received 21 May 2013; Revised 23 July 2013; Accepted 29 July 2013

Academic Editor: Maria Roca

Copyright (C) 2013 Eleonora Cornelia Crasmareanu et al. This is an open access article distributed under the Creative Commons Attribution License, which permits unrestricted use, distribution, and reproduction in any medium, provided the original work is properly cited.

\begin{abstract}
Reversed phase transfer catalysis (RPTC) was applied to synthesize the Naphthol AS-D pigment. This method consists in the transfer of the aryldiazonium cation 4-nitrobenzenediazonium from aqueous medium into the organic phase (nitrobenzene) in the form of a lipophilic ions pair by the catalyst used (perfluorooctyl potassium sulfonates). In the organic phase the azo-coupling reaction between 4-nitrobenzenediazonium chloride and 3-hydroxy-2-carboxylic acid 2-methyl-anilide (Naphthol AS-D) takes place as coupling component. Using this unconventional method of synthesis, an increase of the reaction rate, combined with a higher purity of the product, was obtained.
\end{abstract}

\section{Introduction}

Organic pigments are traditionally used in the mass colouration of plastics and synthetic fibres, and in surface coatings such as paints and inks, and they have also found use in a number of high technology industries [1].

The original process consisted in the addition of phosphorus trichloride to a molten reaction mixture containing aniline and 2-hydroxy-3-naphthoic acid (beta-oxynaphthoic acid, also known as BONA) to give Naphthol AS in good yield [2].

For naphthol AS pigments (naphthol red pigments), the same chemistry is involved as with monoazo yellow pigments, except that the coupling reaction is carried out with arylides of 2-hydroxy-3-naphthoic acid. The reference compound of Naphthols AS is the anilide of 2-hydroxy-3-naphthoic acid. Substitution in the anilide ring affords a series of Naphthol AS derivatives although only a limited number is commercially recognized $[3,4]$. Besides the typical Phase Transfer Catalysis (PTC) reactions, the PTC technique could be applied to reactions involving electrophilic reactant cations, such aryldiazonium or carbonium and anionic catalyst, in which cationic reactant is continuously transferred from aqueous phase into organic phase in the form of a lipophilic ion pair, nonnucleophilic anionic catalyst, and reacts with second reactant from organic phase $[5,6]$. This type of technique was called reversed phase transfer catalysis [7]. The coupling reactions of 4-nitrobenzenediazonium chloride with $\mathrm{N}$-ethylcarbazole and N,N-dimethylaniline in $\mathrm{H}_{2} \mathrm{O} / \mathrm{CH}_{2} \mathrm{Cl}_{2}$ system using sodium dodecylbenzenesulfonate were studied by Ellwood et al. [8]. The same coupling reaction was studied by Iwamoto et al. [9] when sodium tetrakis-[3,5-bis (trifluoromethyl)-phenyl]borate was used as catalyst.

Also Friedel-Crafts type alkylation reactions of carbonium cations with $m$-methylanisole, $o$-cresol, and $m$-dimethoxybenzene were investigated. The carbonium cations were generated in situ by protonation of triphenylmethanol, diphenylmethanol, $p$-methoxybenzyl alcohol, and a-methylbenzyl alcohol [10].

The conventional synthesis method to obtain this type of pigments is based on arylamides of AS-Naphthols. It uses azo-coupling reactions in alcoholic alkaline solutions. The increased $\mathrm{pH}$ favors hydrolysis of amidic group. To avoid this side reaction, $\mathrm{pH}$ must be near to neutral. Also in order to 
increase the solubility of arylamide, the reaction temperature is usually around $60^{\circ} \mathrm{C}$. This can lead to the degradation of the diazonium salt [8].

In this paper we present the synthesis of Naphthol AS pigment by reversed phase transfer catalysis, which is an unconventional method. The pigment was characterized by melting point, TLC, UV-Vis, IR, and mass spectrometry.

\section{Experimental}

2.1. Materials. The solvents nitrobenzene, 1,2-dichloroethane, and toluene (received from Chimopar Bucharest) and the reagents p-Nitroaniline, $\mathrm{NaNO}_{2}$, and $\mathrm{HCl} 37 \%$ were used as received.

The catalyst perfluorooctyl potassium sulfonates $\left(\mathrm{PFOS}^{-} \mathrm{K}^{+}\right.$) were purchased from Sigma-Aldrich.

2.2. Synthesis Method. A mixture of $0.133 \mathrm{~g}\left(7.22 \times 10^{-4} \mathrm{~mol}\right)$ 4-nitrobenzenediazonium chloride in $3 \mathrm{~mL}$ water, $0.1 \mathrm{~g}(3.6 \times$ $10^{-4} \mathrm{~mol}$ ) 3-hydroxy-2-carboxylic acid 2-methyl-anilide in $12 \mathrm{~mL}$ nitrobenzene, and $0.01 \mathrm{~g}$ perfluorooctyl potassium sulfonates $\left(\mathrm{PFOS}^{-} \mathrm{K}^{+}\right.$) as catalyst was used to synthesize Naphthol AS-D pigment by reversed phase transfer catalysis (RPTC) method.

2.3. Spectra Recording. UV-Vis spectra were recorded on a CECIL CE 7200 Spectrophotometer using dimethylformamide solutions of the precursors and products.

FT-IR spectra were recorded on a Jasco FT-IR 4200 spectrometer, using $\mathrm{KBr}$ tablets. The melting point was determined using Boetius Apparatus.

Mass spectra were obtained using a mass spectrometer Esquire 6000 ESI (electrospray ionization) from BrukerDaltonics. The compound was diluted before measurements at $5 \mathrm{pmol} / \mu \mathrm{L}$ in acetonitrile containing $5 \%$ ammonia and was injected in electrospray chamber by direct infusion, with a constant flow of $240 \mu \mathrm{L} / \mathrm{h}$. The mass spectra were obtained in the positive and in the negative modes.

\section{Results and Discussion}

Naphthol AS-D pigment (Figure 1) was synthesized in our laboratory by reversed phase transfer catalysis (RPTC) method.

RPTC method consists in the transfer of the 4-nitrobenzenediazonium $\left(\left[\mathrm{O}_{2} \mathrm{~N}-\mathrm{C}_{6} \mathrm{H}_{4}-\mathrm{N} \equiv \mathrm{N}\right]^{+}\right)$cation, from aqueous medium into the organic phase (nitrobenzene), where the azo-coupling reaction with 3-hydroxy-2-carboxylic acid 2-methyl-anilide (Naphthol AS-D) takes place as coupling component (Figure 2).

The mixture described in experimental section, was vigorously stirred at room temperature. The color development was very fast and the reaction was stopped when the coupling component was consumed. The reaction was monitored using an aryl-diazonium salt solution on filter paper. During the reaction the dyes precipitated and were isolated by filtration. The yield of this synthesis was $98 \%$.

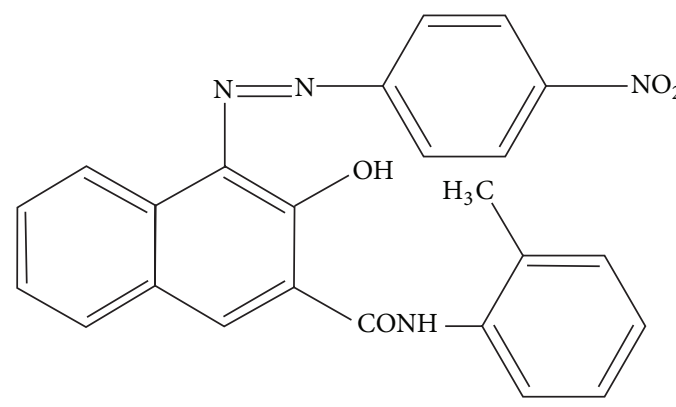

Figure 1: Structure of Naphthol AS-D pigment.

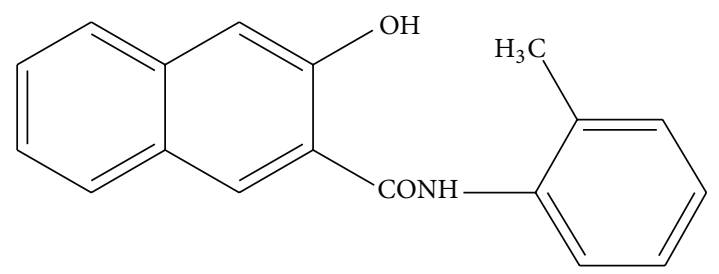

FIgURE 2: Structure of 3-hydroxy-2-carboxylic acid 2-methylanilide (Naphthol AS-D).

The influence on the coupling reaction of the ratio water : nitrobenzene and aryl-diazonium salt : coupling component was tested. The optimum ratio water: nitrobenzene was found at $1: 4$. The molar ratio aryl-diazonium salt: coupling component was $2: 1$ (the standard molar ratio aryl-diazonium salt: coupling component is $1: 1$ ) and the catalyst quantity was kept constant. Other organic phases were tried as well (toluene, 1,2-dichloroethane).

In toluene the reaction proceeded at a lower rate than in nitrobenzene. The formed dye is soluble in dichloroethane, and because of this the separation of the product was more difficult. The coupling reaction was also performed in a biphasic system, in the absence of perfluorooctyl potassium sulfonates. In the reaction mixture an orange color gradually appeared. This indicated that the dye was formed. The purity of dyes was verified by TLC on precoated Kieselgel plates using as eluent mixture benzene:acetone $24: 1\left(R_{f}=0.68\right)$.

The synthesized compound was characterized by melting point, thin layer chromatography (TLC), UV-Vis spectra, FTIR spectra (Figure 3), and mass spectrometry spectra (Figures 4 and 5).

The melting point for Naphthol AS-D pigmentis around $196-200^{\circ} \mathrm{C}$ and its absorption maximum is $505 \mathrm{~nm}$. For performing mass spectra the pigment was diluted before performing the measurement at $5 \mathrm{pmol} / \mu \mathrm{L}$ in acetonitrile.

In the IR spectra (Figure 3) of the obtained pigment the following intense bands of absorption were observed: $\nu_{(\mathrm{N}=\mathrm{N})}=1454 \mathrm{~cm}^{-1}, \nu_{(\mathrm{N}=\mathrm{N})}=1502 \mathrm{~cm}^{-1}$ characteristic for the azo group, $\nu_{(\mathrm{C}=\mathrm{O})}=1680 \mathrm{~cm}^{-1}$ characteristic for the carbonamidic group, $\nu_{(\mathrm{O}-\mathrm{H})}=3629 \mathrm{~cm}^{-1}, \nu_{(\mathrm{C}-\mathrm{N})}=1342 \mathrm{~cm}^{-1}$, $\nu_{\left(\text {aromatic- } \mathrm{CH}_{3}\right)}=3049 \mathrm{~cm}^{-1}, \nu_{\left(\text {aromatic }-\mathrm{NO}_{2}\right)}=1550 \mathrm{~cm}^{-1}$.

The mass spectra obtained for this compound presented better results in the negative mode. Moreover, at these 


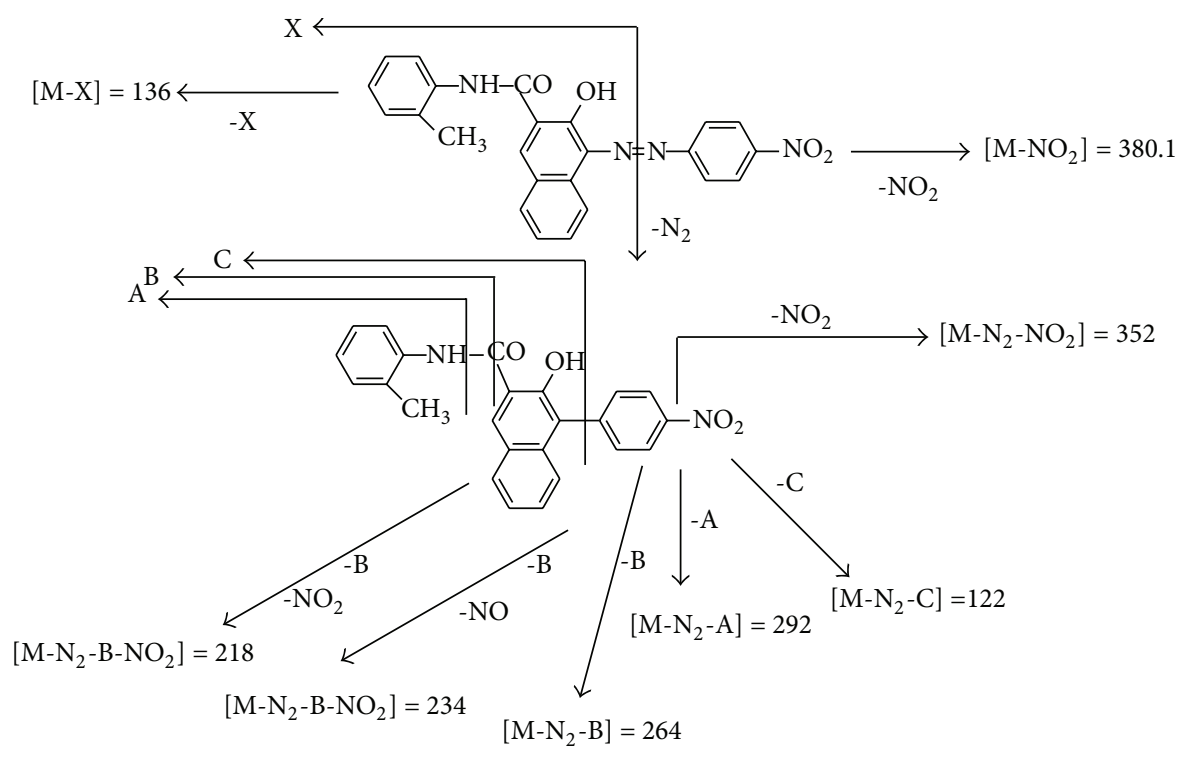

Scheme 1: The structure of the analyzed compound and the fragmentation scheme of Naphthol AS-D Pigment by MS method.

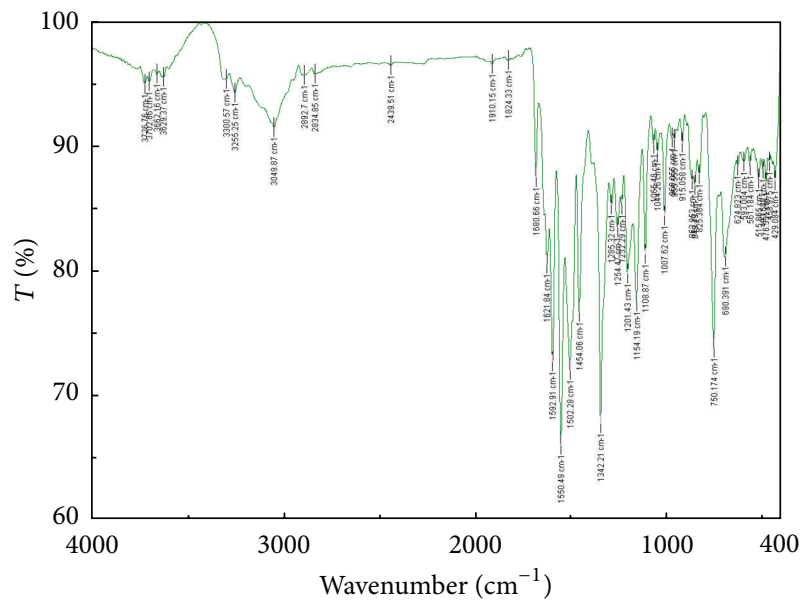

FIGURE 3: IR spectra for Naphthol AS-D pigment.

concentrations it was possible to obtain $\mathrm{MS}^{2}$ spectra with the used ESI source.

The MS spectrum in the negative mode (Figure 4) presented the molecular peak at $\mathrm{m} / z 425$.

The fragmentation of the molecular peak represents the $\mathrm{MS}^{2}$ spectrum (Figure 5).

From $\mathrm{MS}^{2}$ spectrum several significant fragments were observed, of the quasimolecular peak $(\mathrm{m} / z$ 425).

The presence of the peak at $m / z 136$ in $\mathrm{MS}^{2}$ spectrum may be attributed to the rupture of the $\mathrm{N}=\mathrm{N}$ bond. It was also shown that the ion $\mathrm{m} / z 425$ could lose the $\mathrm{NO}_{2}$ group. Further, few peaks showed other fragmentations of the initial molecules, as follow, (Scheme 1):

(i) $\left[\mathrm{M}-\mathrm{N}_{2}-\mathrm{NO}_{2}\right]=m / z 352$,

(ii) $\left[\mathrm{M}-\mathrm{N}_{2}-\mathrm{C}\right]=m / z 122$,

(iii) $\left[\mathrm{M}-\mathrm{N}_{2}-\mathrm{A}\right]=m / z 292$,

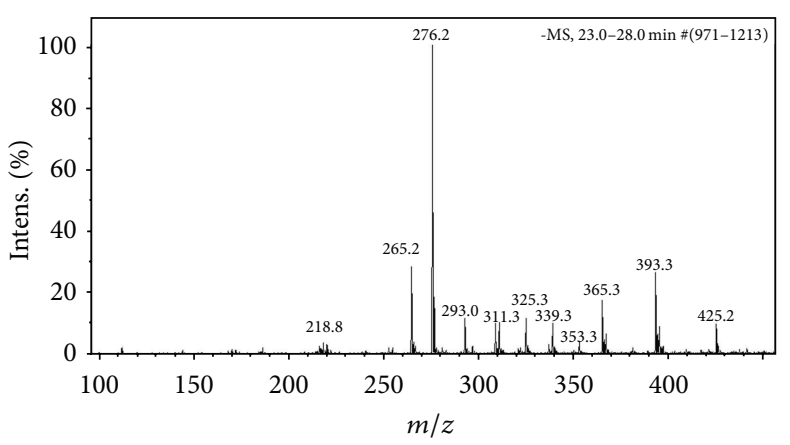

FIGURE 4: ESI-MS spectrum of the Naphthol AS-D pigment in the negative mode.

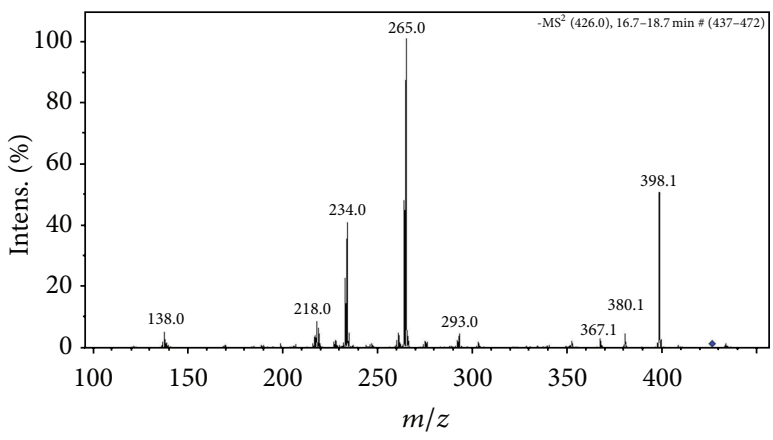

Figure 5: $\mathrm{MS}^{2}$ spectrum of the molecular ion $\mathrm{m} / \mathrm{z} 425$.

(iv) $\left[\mathrm{M}-\mathrm{N}_{2}-\mathrm{B}-\mathrm{NO}\right]=m / z 234$,

(v) $\left[\mathrm{M}-\mathrm{N}_{2}-\mathrm{B}-\mathrm{NO}_{2}\right]=m / z 218$.

The perfluorooctyl potassium sulfonates $\left(\mathrm{PFOS}^{-} \mathrm{K}^{+}\right.$) were found to be essential for the reaction. This may be assumed to the fact that the PFOS anion associates with the 4nitrobenzenediazonium cation and transports it into organic 


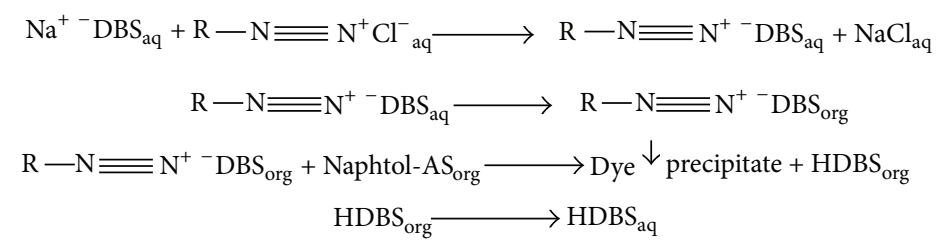

SCHEME 2: RPTC mechanism for obtaining Naphthol AS-D pigment.

phase. The reactivity of the aryl-diazonium cation was increased by the minimization of solvent interactions and the coupling reaction takes place. The liberated PFOS anion can transport more aryl-diazonium ions into the organic phase and thus may be regarded as phase transfer catalyst (Scheme 2) [11, 12].

\section{Conclusions}

All of these results shown in Figures 4 and 5 and in Scheme 1 proved that the assumed structure of Naphthol AS$\mathrm{D}$ pigment was present in the analyzed solution and the synthesis of it was correct. According to the mass spectra, the ionization process was very good in acetonitrile, at the used concentration $(5 \mathrm{pmol} / \mu \mathrm{L})$, in the ESI source. All the observed ions in the mass spectra presented in this paper were singly charged, so the $m / z$ values in all cases were in fact the molecular mass values.

\section{Acknowledgments}

This paper is a result of the project "PhD scholarshipsa key component of excellence research." This project is cofunded by the European Social Fund through The Sectorial Operational Programme for Human Resources Development 2007-2013, coordinated by the West University of Timisoara in partnership with the University of Craiova and Fraunhofer Institute for Integrated Systems and Device TechnologyFraunhofer IISB.

\section{References}

[1] Z. Hao and A. Iqbal, "Some aspects of organic pigments," Chemical Society Reviews, vol. 26, pp. 203-216, 1997.

[2] W. Herbst, K. Hunger, G. Wilker, H. Ohleier, and R. Winter, Industrial Organic Pigments: Production, Properties, Applications, John Wiley \& Sons, Weinheim, Germany, 3rd edition, 2004.

[3] D. Kobelt, E. F. Paulus, and W. Kunstmann, "Röntgeneinkristallstrukturanalyse von 1-(2,5-dichlorphenylazo)-2-hydroxy-3naphthoesäure-(4-chlor-2,5-dimethoxy-anilid) (Chlorderivat von Permanentbraun FG)," Acta Crystallographica B, vol. 28, pp. 1319-1324, 1972.

[4] D. Kobelt, E. F. Paulus, and W. Kunstmann, "Röntgeneinkristallstrukturanalyse eines chlorderivates von permanentrot FRLL. Vergleich mit dem entsprechenden derivat von permanentbraun FG," Zeitschrift Für Kristallographie, vol. 139, pp. 15-32, 1974.
[5] F. Xuening, Z. Tianyong, and Z. Chunlong, "Synthesis of derivatives of Naphtol AS containing polar groups and modification used for C.I. Pigment Red 57," Dyes and Pigments, vol. 40, no. 2-3, pp. 199-204, 1999.

[6] Analytical Chemistry of Synthetic Colorants, A. T. Peters and H. S. Freeman, Eds., vol. 2 of Advances in Color Chemistry Series, pp. 96-116, Chapman \& Hall, London, UK, 1995.

[7] J. J. Jwo, "Phase transfer catalysis: fundamentals and selected systems," Catalysis Reviews, vol. 45, pp. 397-461, 2003.

[8] M. Ellwood, J. Griffiths, and P. Gregory, "First examples of phase transfer catalysis in electrophilic substitution reactions. Acceleration of the azo coupling reaction," Journal of the Chemical Society, Chemical Communications, no. 4, pp. 181-183, 1980.

[9] H. Iwamoto, T. Sonoda, and H. Kobayashi, "A facile in-situ diazo-coupling reactions under two-phase conditions catalyzed by tetrakis[3,5-bis(trifluoromethyl)phenyl]borate ion," Tetrahedron Letters, vol. 24, no. 43, pp. 4703-4706, 1983.

[10] H. Kobayashi, T. Sonoda, and H. Iwamoto, “The first application of anion-catalyzed phase-transfer catalysis to friedel-crafts alkylation," Chemistry Letters, vol. 11, pp. 1185-1186, 1982.

[11] B. F. N. Engbeerts, "Catalysis by surfactant aggregates in aqueous solutions," Pure and Applied Chemistry, vol. 64, pp. 1653-1660, 1992.

[12] MicellarCatalysis, M. N. Khan, Ed., pp. 379-3392, CRC \& Taylor \& Francis, New York, NY, USA, 2006. 

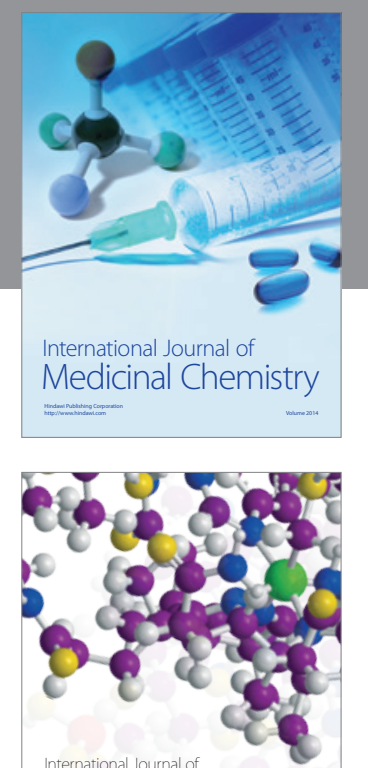

\section{Carbohydrate} Chemistry

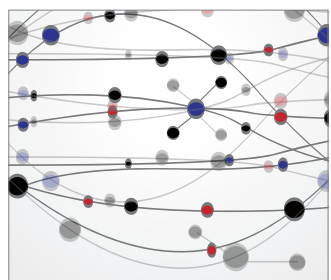

The Scientific World Journal
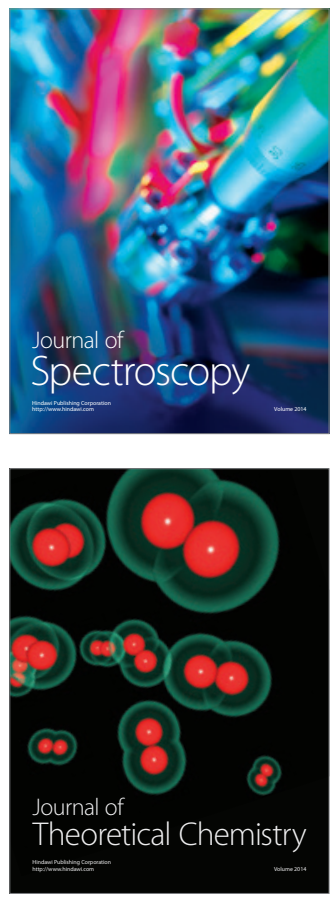
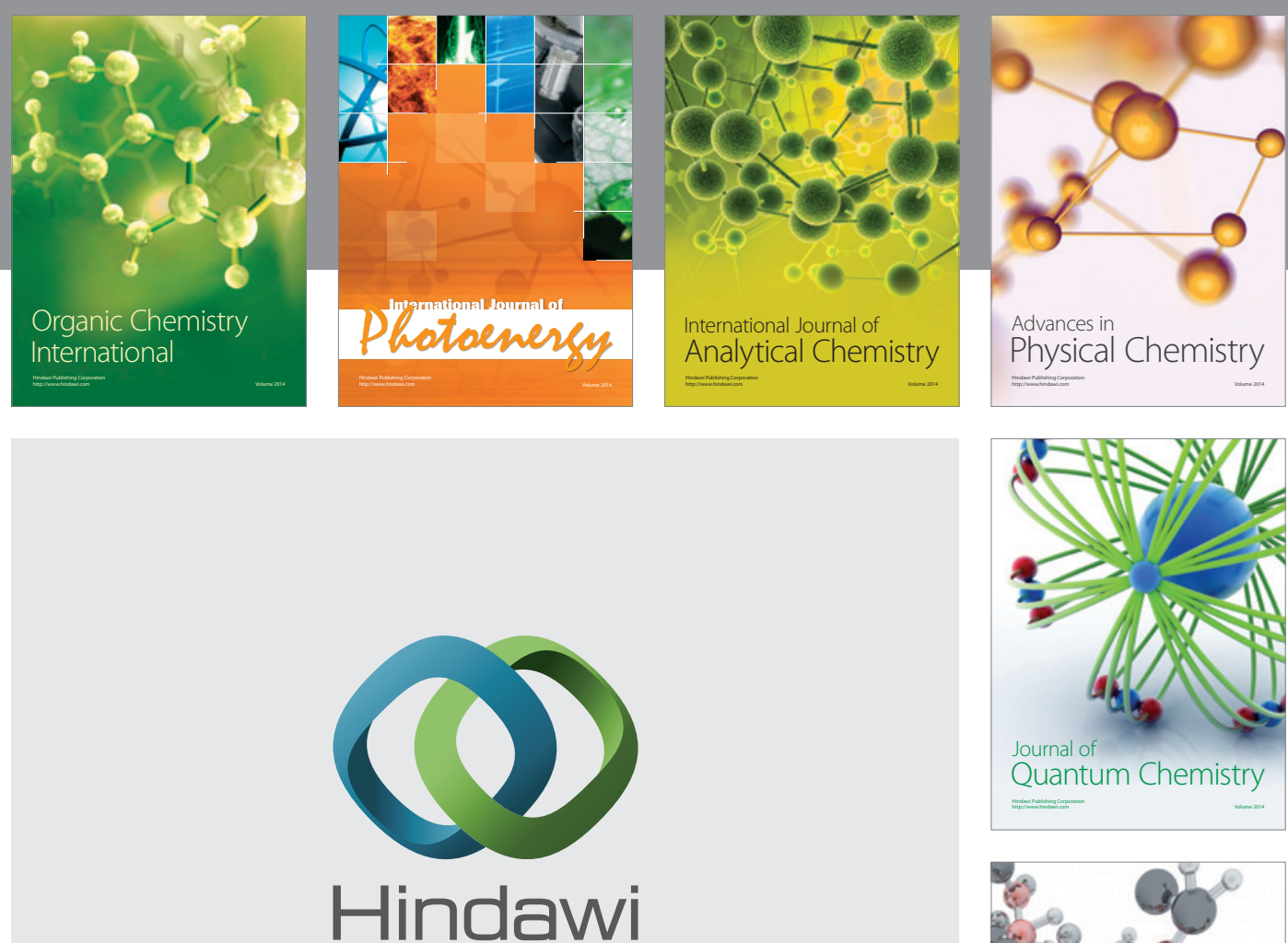

Submit your manuscripts at

http://www.hindawi.com

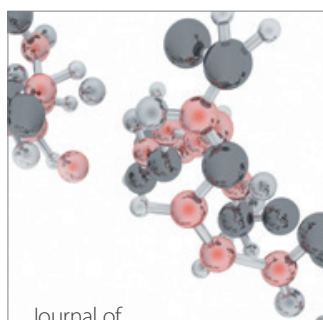

Analytical Methods

in Chemistry

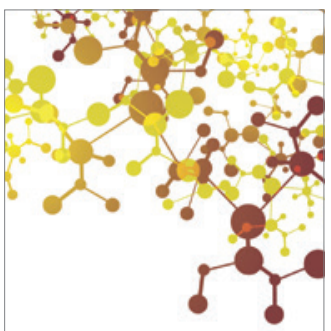

Journal of

Applied Chemistry

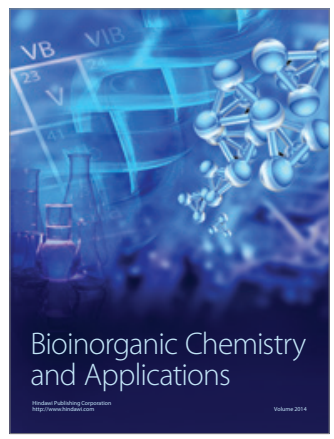

Inorganic Chemistry
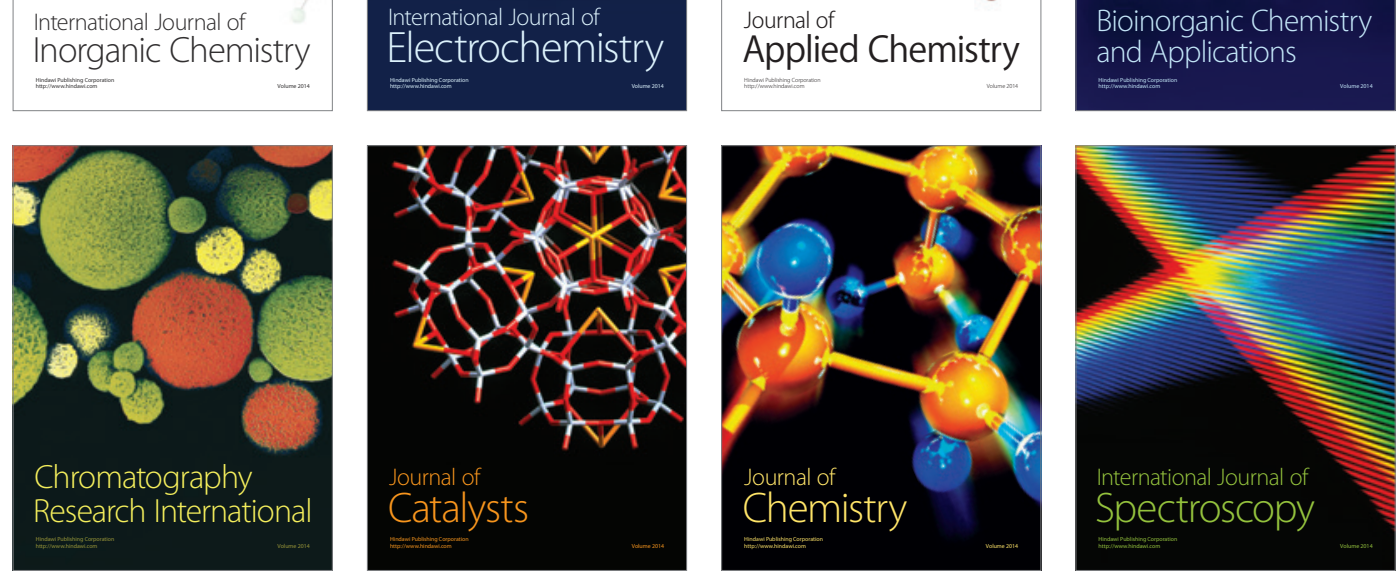